Wei Wang

\title{
Stability of closed characteristics on compact convex hypersurfaces in $\mathbb{R}^{6}$
}

Received April 9, 2007 and in revised form August 3, 2007

Abstract. Let $\Sigma \subset \mathbb{R}^{6}$ be a compact convex hypersurface. We prove that if $\Sigma$ carries only finitely many geometrically distinct closed characteristics, then at least two of them must have irrational mean indices. Moreover, if $\Sigma$ carries exactly three geometrically distinct closed characteristics, then at least two of them must be elliptic.

Keywords. Compact convex hypersurfaces, closed characteristics, Hamiltonian systems, Morse theory, mean index identity, stability

\section{Introduction and main results}

Let $\Sigma$ be a fixed $C^{3}$ compact convex hypersurface in $\mathbb{R}^{2 n}$, i.e., $\Sigma$ is the boundary of a compact and strictly convex region $U$ in $\mathbb{R}^{2 n}$. We denote the set of all such hypersurfaces by $\mathcal{H}(2 n)$. Without loss of generality, we suppose $U$ contains the origin. We consider closed characteristics $(\tau, y)$ on $\Sigma$, which are solutions of the problem

$$
\left\{\begin{array}{l}
\dot{y}=J N_{\Sigma}(y), \\
y(\tau)=y(0),
\end{array} \quad \text { where } \quad J=\left(\begin{array}{cc}
0 & -I_{n} \\
I_{n} & 0
\end{array}\right),\right.
$$

$I_{n}$ is the identity matrix in $\mathbb{R}^{n}, \tau>0, y: \mathbb{R} \rightarrow \mathbb{R}^{2 n}$ with $y(\mathbb{R}) \subset \Sigma$, and $N_{\Sigma}(y)$ is the outward normal vector of $\Sigma$ at $y$ normalized by the condition $N_{\Sigma}(y) \cdot y=1$. Here $a \cdot b$ denotes the standard inner product of $a, b \in \mathbb{R}^{2 n}$. A closed characteristic $(\tau, y)$ is prime if $\tau$ is the minimal period of $y$. Two closed characteristics $(\tau, y)$ and $(\sigma, z)$ are geometrically distinct if $y(\mathbb{R}) \neq z(\mathbb{R})$. We denote by $\mathcal{J}(\Sigma)$ and $\mathcal{J}(\Sigma)$ the set of all closed characteristics $(\tau, y)$ on $\Sigma$ with $\tau$ being the minimal period of $y$ and the set of all geometrically distinct ones respectively. Note that $\mathcal{J}(\Sigma)=\left\{\theta \cdot y \mid \theta \in S^{1}, y\right.$ is prime $\}$, while $\widetilde{\mathcal{J}}(\Sigma)=\mathcal{J}(\Sigma) / S^{1}$, where the natural $S^{1}$-action is defined by $\theta \cdot y(t)=y(t+\tau \theta)$ for $\theta \in S^{1}$ and $t \in \mathbb{R}$.

Let $j: \mathbb{R}^{2 n} \rightarrow \mathbb{R}$ be the gauge function of $\Sigma$, i.e., $j(\lambda x)=\lambda$ for $x \in \Sigma$ and $\lambda \geq 0$, $j \in C^{3}\left(\mathbb{R}^{2 n} \backslash\{0\}, \mathbb{R}\right) \cap C^{0}\left(\mathbb{R}^{2 n}, \mathbb{R}\right)$ and $\Sigma=j^{-1}(1)$. Fix a constant $\alpha \in(1,2)$ and define

W. Wang: School of Mathematical Science, Peking University, Beijing 100871, People's Republic of China; e-mail: alexanderweiwang @yahoo.com.cn

Mathematics Subject Classification (2000): 58E05, 37J45, 37C75 
the Hamiltonian function $H_{\alpha}: \mathbb{R}^{2 n} \rightarrow[0, \infty)$ by

$$
H_{\alpha}(x)=j(x)^{\alpha}, \quad \forall x \in \mathbb{R}^{2 n} .
$$

Then $H_{\alpha} \in C^{3}\left(\mathbb{R}^{2 n} \backslash\{0\}, \mathbb{R}\right) \cap C^{1}\left(\mathbb{R}^{2 n}, \mathbb{R}\right)$ is convex and $\Sigma=H_{\alpha}^{-1}(1)$. It is well known that the problem $(1.1)$ is equivalent to the following given energy problem for the Hamiltonian system:

$$
\left\{\begin{array}{l}
\dot{y}(t)=J H_{\alpha}^{\prime}(y(t)), \quad H_{\alpha}(y(t))=1, \quad \forall t \in \mathbb{R}, \\
y(\tau)=y(0)
\end{array}\right.
$$

Denote by $\mathcal{J}(\Sigma, \alpha)$ the set of all solutions $(\tau, y)$ of $(1.3)$ where $\tau$ is the minimal period of $y$ and by $\widetilde{\mathcal{J}}(\Sigma, \alpha)$ the set of all geometrically distinct solutions of 1.3 . As above, $\widetilde{\mathcal{J}}(\Sigma, \alpha)$ is obtained from $\mathcal{J}(\Sigma, \alpha)$ by dividing by the natural $S^{1}$-action. Note that elements in $\mathcal{J}(\Sigma)$ and $\mathcal{J}(\Sigma, \alpha)$ are in one-to-one correspondence, and similarly for $\widetilde{\mathcal{J}}(\Sigma)$ and $\tilde{\mathcal{J}}(\Sigma, \alpha)$.

Let $(\tau, y) \in \mathcal{J}(\Sigma, \alpha)$. The fundamental solution $\gamma_{y}:[0, \tau] \rightarrow \operatorname{Sp}(2 n)$ with $\gamma_{y}(0)=$ $I_{2 n}$ of the linearized Hamiltonian system

$$
\dot{w}(t)=J H_{\alpha}^{\prime \prime}(y(t)) w(t), \quad \forall t \in \mathbb{R},
$$

is called the associate symplectic path of $(\tau, y)$. The eigenvalues of $\gamma_{y}(\tau)$ are called the Floquet multipliers of $(\tau, y)$. By Proposition 1.6.13 of [Eke3], the Floquet multipliers of $(\tau, y) \in \mathcal{J}(\Sigma)$ together with their multiplicities do not depend on the particular choice of the Hamiltonian function in $(1.3)$. For any $M \in \operatorname{Sp}(2 n)$, we define the elliptic height $e(M)$ of $M$ to be the total algebraic multiplicity of all eigenvalues of $M$ on the unit circle $\mathbb{U}=\{z \in \mathbb{C}|| z \mid=1\}$ in the complex plane $\mathbb{C}$. Since $M$ is symplectic, $e(M)$ is even and $0 \leq e(M) \leq 2 n$. As usual, $(\tau, y) \in \mathcal{J}(\Sigma)$ is elliptic if $e\left(\gamma_{y}(\tau)\right)=2 n$. It is nondegenerate if 1 is a double Floquet multiplier of it, and hyperbolic if 1 is a double Floquet multiplier of it and $e\left(\gamma_{y}(\tau)\right)=2$. It is well known that these concepts are independent of the choice of $\alpha>1$.

For the existence and multiplicity of geometrically distinct closed characteristics on convex compact hypersurfaces in $\mathbb{R}^{2 n}$ we refer to [ [Rab], [Wei], [EkL], [EkH], [Szu], [HWZ], [LoZ], [LLZ], and the references therein. Note that recently in [WHL], Wang, $\mathrm{Hu}$ and Long proved $\# \tilde{\mathcal{J}}(\Sigma) \geq 3$ for every $\Sigma \in \mathcal{H}(6)$.

Concerning the stability problem, Ekeland [Eke2] in 1986 and Long [Lon2] in 1998 proved, for any $\Sigma \in \mathcal{H}(2 n)$, the existence of at least one non-hyperbolic closed characteristic on $\Sigma$ provided $\# \tilde{\mathcal{J}}(\Sigma)<\infty$. Ekeland [Eke2] also proved the existence of at least one elliptic closed characteristic on $\Sigma$ provided $\Sigma \in \mathcal{H}(2 n)$ is $\sqrt{2}$-pinched. In 1992, Dell'Antonio, D'Onofrio and Ekeland [DDE] proved the existence of at least one elliptic closed characteristic on $\Sigma$ provided $\Sigma \in \mathcal{H}(2 n)$ satisfies $\Sigma=-\Sigma$. In 2000, Long [Lon3] proved that $\Sigma \in \mathcal{H}(4)$ and $\# \tilde{\mathcal{J}}(\Sigma)=2$ imply that both the closed characteristics must be elliptic. In 2002, Long [LoZ] and Zhu further proved that when $\# \tilde{\mathcal{J}}(\Sigma)<\infty$, there exists at least one elliptic closed characteristic and there are at least [ $n / 2]$ geometrically distinct closed characteristics on $\Sigma$ possessing irrational mean indices, which are then nonhyperbolic. In the recent paper [LoW], Long and Wang proved that there exist at least 
two nonhyperbolic closed characteristics on $\Sigma \in \mathcal{H}(6)$ when $\# \tilde{\mathcal{J}}(\Sigma)<\infty$. Motivated by these results, we prove the following results:

Theorem 1.1. On every $\Sigma \in \mathcal{H}(6)$ satisfying $\# \tilde{\mathcal{J}}(\Sigma)<\infty$, there exist at least two geometrically distinct closed characteristics with irrational mean indices.

Theorem 1.2. Suppose $\# \tilde{\mathcal{J}}(\Sigma)=3$ for some $\Sigma \in \mathcal{H}(6)$. Then there exist at least two elliptic closed characteristics in $\tilde{\mathcal{J}}(\Sigma)$.

The proofs of Theorems 1.1 and 1.2 are given in Section 3. The main ingredients in the proofs are: the mean index identity for closed characteristics established in [WHL], the Morse inequality and the index iteration theory developed by Long and his coworkers, specially the common index jump theorem of Long and Zhu ([LoZ, Theorem 4.3], cf. [Lon4, Theorem 11.2.1]). In Section 2, we briefly review the equivariant Morse theory and the mean index identity for closed characteristics on compact convex hypersurfaces in $\mathbb{R}^{2 n}$ developed in [WHL].

In this paper, $\mathbb{N}, \mathbb{N}_{0}, \mathbb{Z}, \mathbb{Q}, \mathbb{R}$, and $\mathbb{R}^{+}$denote the sets of natural integers, non-negative integers, integers, rational numbers, real numbers, and positive real numbers respectively. Denote by $a \cdot b$ and $|a|$ the standard inner product and norm in $\mathbb{R}^{2 n}$. Denote by $\langle\cdot, \cdot \cdot\rangle$ and $\|\cdot\|$ the standard $L^{2}$-inner product and $L^{2}$-norm. For an $S^{1}$-space $X$, we denote by $X_{S^{1}}$ the homotopy quotient of $X$ modulo the $S^{1}$-action, i.e., $X_{S^{1}}=S^{\infty} \times_{S^{1}} X$. We define the functions

$[a]=\max \{k \in \mathbb{Z} \mid k \leq a\}, \quad E(a)=\min \{k \in \mathbb{Z} \mid k \geq a\}, \quad \varphi(a)=E(a)-[a]$.

Specifically, $\varphi(a)=0$ if $a \in \mathbb{Z}$, and $\varphi(a)=1$ if $a \notin \mathbb{Z}$. In this paper we use only $\mathbb{Q}$ coefficients for all homology modules. For a $\mathbb{Z}_{m}$-space pair $(A, B)$, let $H_{*}(A, B)^{ \pm \mathbb{Z}_{m}}=$ $\left\{\sigma \in H_{*}(A, B) \mid L_{*} \sigma= \pm \sigma\right\}$, where $L$ is a generator of the $\mathbb{Z}_{m}$-action.

\section{Equivariant Morse theory for closed characteristics}

In the rest of this paper, we fix a $\Sigma \in \mathcal{H}(2 n)$ and assume the following condition on $\Sigma$ :

(F) There exist only finitely many geometrically distinct closed characteristics $\left\{\left(\tau_{j}, y_{j}\right)\right\}_{1 \leq j \leq k}$ on $\Sigma$.

In this section, we briefly review the equivariant Morse theory for closed characteristics on $\Sigma$ developed in [WHL] which will be needed in Section 3. All the details of proofs can be found in [WHL].

Let $\hat{\tau}=\inf \left\{\tau_{j} \mid 1 \leq j \leq k\right\}$. Note that here $\tau_{j}$ 's are prime periods of $y_{j}$ 's for $1 \leq j \leq k$. Then by $\S 2$ of [WHL], for any $a>\hat{\tau}$, we can construct a function $\varphi_{a} \in C^{\infty}\left(\mathbb{R}, \mathbb{R}^{+}\right)$such that 0 is the unique critical point in $[0, \infty)$ and $\varphi_{a}$ is strictly convex for $t \geq 0$. Moreover, $\varphi_{a}^{\prime}(t) / t$ is strictly decreasing for $t>0$ with $\lim _{t \rightarrow 0^{+}} \varphi_{a}^{\prime}(t) / t=1$ and $\varphi_{a}(0)=0=$ $\varphi_{a}^{\prime}(0)$. More precisely, we define $\varphi_{a}$ via Propositions 2.2 and 2.4 of [WHL]. The precise dependence of $\varphi_{a}$ on $a$ is explained in Remark 2.3 of [WHL]. 
Define the Hamiltonian function $H_{a}(x)=a \varphi_{a}(j(x))$ and consider the fixed period problem

$$
\left\{\begin{array}{l}
\dot{x}(t)=J H_{a}^{\prime}(x(t)) \\
x(1)=x(0)
\end{array}\right.
$$

Then $H_{a} \in C^{3}\left(\mathbb{R}^{2 n} \backslash\{0\}, \mathbb{R}\right) \cap C^{1}\left(\mathbb{R}^{2 n}, \mathbb{R}\right)$ is strictly convex. Solutions of 2.1 are $x \equiv 0$ and $x=\rho y(\tau t)$ with $\varphi_{a}^{\prime}(\rho) / \rho=\tau / a$, where $(\tau, y)$ is a solution of 1.1. In particular, nonzero solutions of 2.1 are in one-to-one correspondence with solutions of [1.1] with period $\tau<a$.

In the following, we use the Clarke-Ekeland dual action principle. As usual, let $G_{a}$ be the Fenchel transform of $H_{a}$ defined by $G_{a}(y)=\sup \left\{x \cdot y-H_{a}(x) \mid x \in \mathbb{R}^{2 n}\right\}$. Then $G_{a} \in C^{2}\left(\mathbb{R}^{2 n} \backslash\{0\}, \mathbb{R}\right) \cap C^{1}\left(\mathbb{R}^{2 n}, \mathbb{R}\right)$ is strictly convex. Let

$$
L_{0}^{2}\left(S^{1}, \mathbb{R}^{2 n}\right)=\left\{u \in L^{2}\left([0,1], \mathbb{R}^{2 n}\right) \mid \int_{0}^{1} u(t) d t=0\right\} .
$$

Define a linear operator $M: L_{0}^{2}\left(S^{1}, \mathbb{R}^{2 n}\right) \rightarrow L_{0}^{2}\left(S^{1}, \mathbb{R}^{2 n}\right)$ by $\frac{d}{d t} M u(t)=u(t)$ and $\int_{0}^{1} M u(t) d t=0$. The dual action functional on $L_{0}^{2}\left(S^{1}, \mathbb{R}^{2 n}\right)$ is defined by

$$
\Psi_{a}(u)=\int_{0}^{1}\left(\frac{1}{2} J u \cdot M u+G_{a}(-J u)\right) d t .
$$

Then the functional $\Psi_{a} \in C^{1,1}\left(L_{0}^{2}\left(S^{1}, \mathbb{R}^{2 n}\right), \mathbb{R}\right)$ is bounded below and satisfies the Palais-Smale condition. Suppose $x$ is a solution of 2.1). Then $u=\dot{x}$ is a critical point of $\Psi_{a}$. Conversely, suppose $u$ is a critical point of $\Psi_{a}$. Then there exists a unique $\xi \in \mathbb{R}^{2 n}$ such that $M u-\xi$ is a solution of 2.1). In particular, solutions of (2.1) are in one-to-one correspondence with critical points of $\Psi_{a}$. Moreover, $\Psi_{a}(u)<0$ for every critical point $u \neq 0$ of $\Psi_{a}$.

Suppose $u$ is a nonzero critical point of $\Psi_{a}$. Then following [Eke3] the formal Hessian of $\Psi_{a}$ at $u$ is defined by

$$
Q_{a}(v, v)=\int_{0}^{1}\left(J v \cdot M v+G_{a}^{\prime \prime}(-J u) J v \cdot J v\right) d t,
$$

which defines an orthogonal splitting $L_{0}^{2}=E_{-} \oplus E_{0} \oplus E_{+}$of $L_{0}^{2}\left(S^{1}, \mathbb{R}^{2 n}\right)$ into the negative, zero and positive subspaces. The index of $u$ is defined by $i(u)=\operatorname{dim} E_{-}$, and the nullity of $u$ is $v(u)=\operatorname{dim} E_{0}$. Let $u=\dot{x}$ be the critical point of $\Psi_{a}$ such that $x$ corresponds to the closed characteristic $(\tau, y)$ on $\Sigma$. Then the index $i(u)$ and the nullity $v(u)$ defined above coincide with the Ekeland indices defined in [Eke1] and [Eke3]. In paticular, $1 \leq v(u) \leq 2 n-1$ always holds.

We have a natural $S^{1}$-action on $L_{0}^{2}\left(S^{1}, \mathbb{R}^{2 n}\right)$ defined by $\theta \cdot u(t)=u(\theta+t)$ for all $\theta \in S^{1}$ and $t \in \mathbb{R}$. Clearly $\Psi_{a}$ is $S^{1}$-invariant. For any $\kappa \in \mathbb{R}$, we define

$$
\Lambda_{a}^{\kappa}=\left\{u \in L_{0}^{2}\left(S^{1}, \mathbb{R}^{2 n}\right) \mid \Psi_{a}(u) \leq \kappa\right\} .
$$


For a critical point $u$ of $\Psi_{a}$, we set

$$
\Lambda_{a}(u)=\Lambda_{a}^{\Psi_{a}(u)}=\left\{w \in L_{0}^{2}\left(S^{1}, \mathbb{R}^{2 n}\right) \mid \Psi_{a}(w) \leq \Psi_{a}(u)\right\} .
$$

Clearly, both sets are $S^{1}$-invariant. Since the $S^{1}$-action preserves $\Psi_{a}$, if $u$ is a critical point of $\Psi_{a}$, then the whole orbit $S^{1} \cdot u$ is formed by critical points of $\Psi_{a}$. Denote by $\operatorname{crit}\left(\Psi_{a}\right)$ the set of critical points of $\Psi_{a}$. Note that by the condition $(\mathrm{F})$, the number of critical orbits of $\Psi_{a}$ is finite. Hence as usual we can make the following definition.

Definition 2.1. Suppose $u$ is a nonzero critical point of $\Psi_{a}$ and $\mathcal{N}$ is an $S^{1}$-invariant open neighborhood of $S^{1} \cdot u$ such that $\operatorname{crit}\left(\Psi_{a}\right) \cap\left(\Lambda_{a}(u) \cap \mathcal{N}\right)=S^{1} \cdot u$. Then the $S^{1}$-critical modules of $S^{1} \cdot u$ are defined by

$$
C_{S^{1}, q}\left(\Psi_{a}, S^{1} \cdot u\right)=H_{q}\left(\left(\Lambda_{a}(u) \cap \mathcal{N}\right)_{S^{1}},\left(\left(\Lambda_{a}(u) \backslash S^{1} \cdot u\right) \cap \mathcal{N}\right)_{S^{1}}\right) .
$$

We have the following proposition for critical modules.

Proposition 2.2 (Proposition 3.2 of [WHL]). The critical module $C_{S^{1}, q}\left(\Psi_{a}, S^{1} \cdot u\right)$ is independent of $a$ in the sense that if $x_{i}$ are solutions of 2.1) with Hamiltonian functions $H_{a_{i}}(x) \equiv a_{i} \varphi_{a_{i}}(j(x))$ for $i=1,2$ respectively such that both $x_{1}$ and $x_{2}$ correspond to the same closed characteristic $(\tau, y)$ on $\Sigma$, then

$$
C_{S^{1}, q}\left(\Psi_{a_{1}}, S^{1} \cdot \dot{x}_{1}\right) \cong C_{S^{1}, q}\left(\Psi_{a_{2}}, S^{1} \cdot \dot{x}_{2}\right), \quad \forall q \in \mathbb{Z} .
$$

Now let $u \neq 0$ be a critical point of $\Psi_{a}$ with multiplicity $\operatorname{mul}(u)=m$, i.e., $u$ corresponds to a closed characteristic $(m \tau, y) \subset \Sigma$ with $(\tau, y)$ being prime. Hence $u(t+1 / m)=u(t)$ for all $t \in \mathbb{R}$ and $S^{1} \cdot u \cong S^{1} / \mathbb{Z}_{m} \cong S^{1}$. Let $f: N\left(S^{1} \cdot u\right) \rightarrow S^{1} \cdot u$ be the normal bundle of $S^{1} \cdot u$ in $L_{0}^{2}\left(S^{1}, \mathbb{R}^{2 n}\right)$ and let $f^{-1}(\theta \cdot u)=N(\theta \cdot u)$ be the fiber over $\theta \cdot u$, where $\theta \in S^{1}$. Let $D N\left(S^{1} \cdot u\right)$ be the $\varrho$-disk bundle of $N\left(S^{1} \cdot u\right)$ for some $\varrho>0$ sufficiently small, i.e., $D N\left(S^{1} \cdot u\right)=\left\{\xi \in N\left(S^{1} \cdot u\right) \mid\|\xi\|<\varrho\right\}$, and let $D N(\theta \cdot u)=f^{-1}(\theta \cdot u) \cap D N\left(S^{1} \cdot u\right)$ be the disk over $\theta \cdot u$. Clearly, $D N(\theta \cdot u)$ is $\mathbb{Z}_{m}$-invariant and we have $D N\left(S^{1} \cdot u\right)=$ $D N(u) \times_{\mathbb{Z}_{m}} S^{1}$, where the $\mathbb{Z}_{m}$-action is given by

$$
(\theta, v, t) \in \mathbb{Z}_{m} \times D N(u) \times S^{1} \mapsto\left(\theta \cdot v, \theta^{-1} t\right) \in D N(u) \times S^{1} .
$$

Hence for an $S^{1}$-invariant subset $\Gamma$ of $D N\left(S^{1} \cdot u\right)$, we have $\Gamma / S^{1}=\left(\Gamma_{u} \times \mathbb{Z}_{m} S^{1}\right) / S^{1}=$ $\Gamma_{u} / \mathbb{Z}_{m}$, where $\Gamma_{u}=\Gamma \cap D N(u)$. Since $\Psi_{a}$ is not $C^{2}$ on $L_{0}^{2}\left(S^{1}, \mathbb{R}^{2 n}\right)$, we need to use a finite-dimensional approximation introduced by Ekeland in order to apply Morse theory. More precisely, we can construct a finite-dimensional submanifold $\Gamma(\iota)$ of $L_{0}^{2}\left(S^{1}, \mathbb{R}^{2 n}\right)$ which admits a $\mathbb{Z}_{\iota}$-action with $m \mid \iota$. Moreover, $\Psi_{a}$ and $\left.\Psi_{a}\right|_{\Gamma(\iota)}$ have the same critical points. $\left.\Psi_{a}\right|_{\Gamma(\iota)}$ is $C^{2}$ in a small tubular neighborhood of the critical orbit $S^{1} \cdot u$, and the Morse index and nullity of its critical points coincide with those of the corresponding critical points of $\Psi_{a}$. Let

$$
D_{\iota} N\left(S^{1} \cdot u\right)=D N\left(S^{1} \cdot u\right) \cap \Gamma(\iota), \quad D_{\iota} N(\theta \cdot u)=D N(\theta \cdot u) \cap \Gamma(\iota) .
$$

Then we have

$$
C_{S^{1}, *}\left(\Psi_{a}, S^{1} \cdot u\right) \cong H_{*}\left(\Lambda_{a}(u) \cap D_{\iota} N(u),\left(\Lambda_{a}(u) \backslash\{u\}\right) \cap D_{\iota} N(u)\right)^{\mathbb{Z}_{m}} .
$$


Now we can apply the results of Gromoll and Meyer [GrM] to the manifold $D_{p l} N\left(u^{p}\right)$ with unique critical point $u^{p}$, where $p \in \mathbb{N}$. Then $\operatorname{mul}\left(u^{p}\right)=p m$ is the multiplicity of $u^{p}$ and the isotropy group $\mathbb{Z}_{p m} \subseteq S^{1}$ of $u^{p}$ acts on $D_{p \iota} N\left(u^{p}\right)$ by isometries. According to Lemma 1 of [GrM], we have a $\mathbb{Z}_{p m}$-invariant decomposition

$$
T_{u} p\left(D_{p l} N\left(u^{p}\right)\right)=V^{+} \oplus V^{-} \oplus V^{0}=\left\{\left(x_{+}, x_{-}, x_{0}\right)\right\}
$$

with $\operatorname{dim} V^{-}=i\left(u^{p}\right), \operatorname{dim} V^{0}=v\left(u^{p}\right)-1$ and a $\mathbb{Z}_{p m}$-invariant neighborhood $B=$ $B_{+} \times B_{-} \times B_{0}$ of 0 in $T_{u} p\left(D_{p l} N\left(u^{p}\right)\right)$ together with two $\mathbb{Z}_{p m}$-invariant diffeomorphisms

$$
\Phi: B=B_{+} \times B_{-} \times B_{0} \rightarrow \Phi\left(B_{+} \times B_{-} \times B_{0}\right) \subset D_{p l} N\left(u^{p}\right)
$$

and

$$
\eta: B_{0} \rightarrow W\left(u^{p}\right) \equiv \eta\left(B_{0}\right) \subset D_{p l} N\left(u^{p}\right)
$$

such that $\Phi(0)=\eta(0)=u^{p}$ and

$$
\Psi_{a} \circ \Phi\left(x_{+}, x_{-}, x_{0}\right)=\left|x_{+}\right|^{2}-\left|x_{-}\right|^{2}+\Psi_{a} \circ \eta\left(x_{0}\right),
$$

with $d\left(\Psi_{a} \circ \eta\right)(0)=d^{2}\left(\Psi_{a} \circ \eta\right)(0)=0$. Following [GrM], we call $W\left(u^{p}\right)$ a local characteristic manifold and $U\left(u^{p}\right)=B_{-}$a local negative disk at $u^{p}$. By the proof of Lemma 1 of [GrM], $W\left(u^{p}\right)$ and $U\left(u^{p}\right)$ are $\mathbb{Z}_{p m}$-invariant. Then we have

$$
\begin{aligned}
& H_{*}\left(\Lambda_{a}\left(u^{p}\right) \cap D_{p l} N\left(u^{p}\right),\left(\Lambda_{a}\left(u^{p}\right) \backslash\left\{u^{p}\right\}\right) \cap D_{p \iota} N\left(u^{p}\right)\right) \\
& =H_{*}\left(U\left(u^{p}\right), U\left(u^{p}\right) \backslash\left\{u^{p}\right\}\right) \otimes H_{*}\left(W\left(u^{p}\right) \cap \Lambda_{a}\left(u^{p}\right),\left(W\left(u^{p}\right) \backslash\left\{u^{p}\right\}\right) \cap \Lambda_{a}\left(u^{p}\right)\right),
\end{aligned}
$$

where

$$
H_{q}\left(U\left(u^{p}\right), U\left(u^{p}\right) \backslash\left\{u^{p}\right\}\right)= \begin{cases}\mathbb{Q} & \text { if } q=i\left(u^{p}\right) \\ 0 & \text { otherwise }\end{cases}
$$

Now we have the following proposition.

Proposition 2.3 (Proposition 3.10 of [WHL]). Let $u \neq 0$ be a critical point of $\Psi_{a}$ with $\operatorname{mul}(u)=1$. Then for all $p \in \mathbb{N}$ and $q \in \mathbb{Z}$, we have

$$
C_{S^{1}, q}\left(\Psi_{a}, S^{1} \cdot u^{p}\right) \cong H_{q-i\left(u^{p}\right)}\left(W\left(u^{p}\right) \cap \Lambda_{a}\left(u^{p}\right),\left(W\left(u^{p}\right) \backslash\left\{u^{p}\right\}\right) \cap \Lambda_{a}\left(u^{p}\right)\right)^{\beta\left(u^{p}\right) \mathbb{Z}_{p}},
$$

where $\beta\left(u^{p}\right)=(-1)^{i\left(u^{p}\right)-i(u)}$. Thus

$$
C_{S^{1}, q}\left(\Psi_{a}, S^{1} \cdot u^{p}\right)=0 \quad \text { if } q<i\left(u^{p}\right) \text { or } q>i\left(u^{p}\right)+v\left(u^{p}\right)-1 .
$$

In particular, if $u^{p}$ is nondegenerate, i.e., $v\left(u^{p}\right)=1$, then

$$
C_{S^{1}, q}\left(\Psi_{a}, S^{1} \cdot u^{p}\right)= \begin{cases}\mathbb{Q} & \text { if } q=i\left(u^{p}\right) \text { and } \beta\left(u^{p}\right)=1, \\ 0 & \text { otherwise. }\end{cases}
$$

We make the following definition: 
Definition 2.4. Let $u \neq 0$ be a critical point of $\Psi_{a}$ with $\operatorname{mul}(u)=1$. Then for all $p \in \mathbb{N}$ and $l \in \mathbb{Z}$, let

$$
\begin{aligned}
k_{l, \pm 1}\left(u^{p}\right) & =\operatorname{dim} H_{l}\left(W\left(u^{p}\right) \cap \Lambda_{a}\left(u^{p}\right),\left(W\left(u^{p}\right) \backslash\left\{u^{p}\right\}\right) \cap \Lambda_{a}\left(u^{p}\right)\right)^{ \pm \mathbb{Z}_{p}}, \\
k_{l}\left(u^{p}\right) & =\operatorname{dim} H_{l}\left(W\left(u^{p}\right) \cap \Lambda_{a}\left(u^{p}\right),\left(W\left(u^{p}\right) \backslash\left\{u^{p}\right\}\right) \cap \Lambda_{a}\left(u^{p}\right)\right)^{\beta\left(u^{p}\right) \mathbb{Z}_{p} .}
\end{aligned}
$$

The $k_{l}\left(u^{p}\right)$ 's are called the critical type numbers of $u^{p}$.

We have the following properties for critical type numbers:

Proposition 2.5 (Proposition 3.13 of [WHL]). Let $u \neq 0$ be a critical point of $\Psi_{a}$ with $\operatorname{mul}(u)=1$. Then there exists a minimal $K(u) \in \mathbb{N}$ such that

$$
v\left(u^{p+K(u)}\right)=v\left(u^{p}\right), \quad i\left(u^{p+K(u)}\right)-i\left(u^{p}\right) \in 2 \mathbb{Z},
$$

and $k_{l}\left(u^{p+K(u)}\right)=k_{l}\left(u^{p}\right)$ for all $p \in \mathbb{N}$ and $l \in \mathbb{Z}$. We call $K(u)$ the minimal period of critical modules of iterations of the functional $\Psi_{a}$ at $u$.

For a closed characteristic $(\tau, y)$ on $\Sigma$, we denote by $y^{m} \equiv(m \tau, y)$ the $m$-th iteration of $y$ for $m \in \mathbb{N}$. Let $a>\tau$ and choose $\varphi_{a}$ as above. Determine $\rho$ uniquely by $\varphi_{a}^{\prime}(\rho) / \rho=\tau / a$. Let $x=\rho y(\tau t)$ and $u=\dot{x}$. Then we define the index $i\left(y^{m}\right)$ and nullity $\nu\left(y^{m}\right)$ of $(m \tau, y)$ for $m \in \mathbb{N}$ by

$$
i\left(y^{m}\right)=i\left(u^{m}\right), \quad v\left(y^{m}\right)=v\left(u^{m}\right) .
$$

These indices are independent of $a$ when $a$ tends to infinity. Now the mean index of $(\tau, y)$ is defined by

$$
\hat{i}(y)=\lim _{m \rightarrow \infty} \frac{i\left(y^{m}\right)}{m} .
$$

Note that always $\hat{i}(y)>2$, which was proved by Ekeland and Hofer [EkH] in 1987 (cf. Corollary 8.3.2 and Lemma 15.3.2 of [Lon4] for a different proof).

By Proposition 2.2, we can define the critical type numbers $k_{l}\left(y^{m}\right)$ of $y^{m}$ to be $k_{l}\left(u^{m}\right)$, where $u^{m}$ is the critical point of $\Psi_{a}$ corresponding to $y^{m}$. We also define $K(y)=K(u)$. Then we have

Proposition 2.6. We have $k_{l}\left(y^{m}\right)=0$ for $l \notin\left[0, v\left(y^{m}\right)-1\right]$ and it can take only values 0 or 1 when $l=0$ or $l=v\left(y^{m}\right)-1$. Moreover, the following properties hold (cf. Lemma 3.10 of $[\overline{\mathrm{BaL}}]$, [Cha] and $[\overline{\mathrm{MaW}}])$ :

(i) $k_{0}\left(y^{m}\right)=1$ implies $k_{l}\left(y^{m}\right)=0$ for $1 \leq l \leq v\left(y^{m}\right)-1$.

(ii) $k_{v\left(y^{m}\right)-1}\left(y^{m}\right)=1$ implies $k_{l}\left(y^{m}\right)=0$ for $0 \leq l \leq v\left(y^{m}\right)-2$.

(iii) $k_{l}\left(y^{m}\right) \geq 1$ for some $1 \leq l \leq v\left(y^{m}\right)-2$ implies $k_{0}\left(y^{m}\right)=k_{v\left(y^{m}\right)-1}\left(y^{m}\right)=0$.

(iv) If $v\left(y^{m}\right) \leq 3$, then at most one of the $k_{l}\left(y^{m}\right)^{\prime}$ 's for $0 \leq l \leq v\left(y^{m}\right)-1$ can be non-zero.

(v) If $i\left(y^{m}\right)-i(y) \in 2 \mathbb{Z}+1$ for some $m \in \mathbb{N}$, then $k_{0}\left(y^{m}\right)=0$. 
Proof. By Definition 2.4 we have

$$
k_{l}\left(y^{m}\right) \leq \operatorname{dim} H_{l}\left(W\left(u^{m}\right) \cap \Lambda_{a}\left(u^{m}\right),\left(W\left(u^{m}\right) \backslash\left\{u^{m}\right\}\right) \cap \Lambda_{a}\left(u^{m}\right)\right) \equiv \eta_{l}\left(y^{m}\right) .
$$

Then from Corollary 1.5.1 of [Cha] or Corollary 8.4 of [MaW], (i)-(iv) hold.

If $\eta_{0}\left(y^{m}\right)=0$, then (v) follows directly from Definition 2.4 .

By Corollary 8.4 of [MaW], $\eta_{0}\left(y^{m}\right)=1$ if and only if $u^{m}$ is a local minimum in the local characteristic manifold $W\left(u^{m}\right)$. Hence $\left(W\left(u^{m}\right) \cap \Lambda_{a}\left(u^{m}\right),\left(W\left(u^{m}\right) \backslash\left\{u^{m}\right\}\right) \cap\right.$ $\left.\Lambda_{a}\left(u^{m}\right)\right)=\left(\left\{u^{m}\right\}, \emptyset\right)$. By Definition 2.4, we have

$$
\begin{aligned}
k_{0,+1}\left(u^{m}\right) & =\operatorname{dim} H_{0}\left(W\left(u^{m}\right) \cap \Lambda_{a}\left(u^{m}\right),\left(W\left(u^{m}\right) \backslash\left\{u^{m}\right\}\right) \cap \Lambda_{a}\left(u^{m}\right)\right)^{+\mathbb{Z}_{m}} \\
& =\operatorname{dim} H_{0}\left(\left\{u^{m}\right\}\right)^{+\mathbb{Z}_{m}}=1 .
\end{aligned}
$$

This implies $k_{0}\left(u^{m}\right)=k_{0,-1}\left(u^{m}\right)=0$.

For a closed characteristic $(\tau, y)$ on $\Sigma$, we define, as in [WHL],

$$
\hat{\chi}(y)=\frac{1}{K(y)} \sum_{\substack{1 \leq m \leq K(y) \\ 0 \leq l \leq 2 n-2}}(-1)^{i\left(y^{m}\right)+l} k_{l}\left(y^{m}\right) .
$$

In particular, if all $y^{m}$ 's are nondegenerate, then by Proposition 2.3 we have

$$
\hat{\chi}(y)= \begin{cases}(-1)^{i(y)} & \text { if } i\left(y^{2}\right)-i(y) \in 2 \mathbb{Z}, \\ (-1)^{i(y)} / 2 & \text { otherwise. }\end{cases}
$$

We have the following mean index identity for closed characteristics.

Theorem 2.7 (Theorem 1.2 of [WHL]). Suppose $\Sigma \in \mathcal{H}(2 n)$ satisfies $\# \tilde{\mathcal{J}}(\Sigma)<\infty$. Let $\left\{\left(\tau_{j}, y_{j}\right)\right\}_{1 \leq j \leq k}$ be all the geometrically distinct closed characteristics. Then

$$
\sum_{1 \leq j \leq k} \frac{\hat{\chi}\left(y_{j}\right)}{\hat{i}\left(y_{j}\right)}=\frac{1}{2}
$$

Let $\Psi_{a}$ be the functional defined by 2.3 for some $a \in \mathbb{R}$ large enough and let $\varepsilon>0$ be small enough such that $[-\varepsilon, \infty) \backslash\{0\}$ contains no critical values of $\Psi_{a}$. Denote by $I_{a}$ the greatest integer in $\mathbb{N}_{0}$ such that $I_{a}<i(\tau, y)$ for all closed characteristics $(\tau, y)$ on $\Sigma$ with $\tau \geq a$. Then by Section 5 of [WHL], we have

$$
H_{S^{1}, q}\left(\Lambda_{a}^{-\varepsilon}\right) \cong H_{S^{1}, q}\left(\Lambda_{a}^{\infty}\right) \cong H_{q}\left(\mathbb{C} P^{\infty}\right), \quad \forall q<I_{a} .
$$

For any $q \in \mathbb{Z}$, let

$$
M_{q}\left(\Lambda_{a}^{-\varepsilon}\right)=\sum_{1 \leq j \leq k, 1 \leq m_{j}<a / \tau_{j}} \operatorname{dim} C_{S^{1}, q}\left(\Psi_{a}, S^{1} \cdot u_{j}^{m_{j}}\right) .
$$


Then the equivariant Morse inequalities for the space $\Lambda_{a}^{-\varepsilon}$ yield

$$
\begin{gathered}
M_{q}\left(\Lambda_{a}^{-\varepsilon}\right) \geq b_{q}\left(\Lambda_{a}^{-\varepsilon}\right), \\
M_{q}\left(\Lambda_{a}^{-\varepsilon}\right)-M_{q-1}\left(\Lambda_{a}^{-\varepsilon}\right)+\cdots+(-1)^{q} M_{0}\left(\Lambda_{a}^{-\varepsilon}\right) \\
\geq b_{q}\left(\Lambda_{a}^{-\varepsilon}\right)-b_{q-1}\left(\Lambda_{a}^{-\varepsilon}\right)+\cdots+(-1)^{q} b_{0}\left(\Lambda_{a}^{-\varepsilon}\right),
\end{gathered}
$$

where $b_{q}\left(\Lambda_{a}^{-\varepsilon}\right)=\operatorname{dim} H_{S^{1}, q}\left(\Lambda_{a}^{-\varepsilon}\right)$. Now we have the following Morse inequalities for closed characteristics:

Theorem 2.8. Let $\Sigma \in \mathcal{H}(2 n)$ satisfy $\# \widetilde{\mathcal{J}}(\Sigma)<\infty$. Let $\left\{\left(\tau_{j}, y_{j}\right)\right\}_{1 \leq j \leq k}$ be all the geometrically distinct closed characteristics. Let

$$
\begin{aligned}
& M_{q}=\lim _{a \rightarrow \infty} M_{q}\left(\Lambda_{a}^{-\varepsilon}\right), \quad \forall q \in \mathbb{Z}, \\
& b_{q}=\lim _{a \rightarrow \infty} b_{q}\left(\Lambda_{a}^{-\varepsilon}\right)= \begin{cases}1 & \text { if } q \in 2 \mathbb{N}_{0}, \\
0 & \text { otherwise }\end{cases}
\end{aligned}
$$

Then

$$
\begin{aligned}
M_{q} & \geq b_{q}, \\
M_{q}-M_{q-1}+\cdots+(-1)^{q} M_{0} & \geq b_{q}-b_{q-1}+\cdots+(-1)^{q} b_{0}, \quad \forall q \in \mathbb{Z} .
\end{aligned}
$$

Proof. As mentioned before, $\hat{i}\left(y_{j}\right)>2$ for $1 \leq j \leq k$. Hence the Ekeland index satisfies $i\left(y_{j}^{m}\right)=i\left(u_{j}^{m}\right) \rightarrow \infty$ as $m \rightarrow \infty$ for $1 \leq j \leq k$. Note that $I_{a} \rightarrow \infty$ as $a \rightarrow \infty$. Now fix a $q \in \mathbb{Z}$ and a sufficiently large $a>0$. By Propositions 2.2, 2.3 and 2.17$), M_{i}\left(\Lambda_{a}^{-\varepsilon}\right)$ is invariant for all $a>A_{q}$ and $0 \leq i \leq q$, where $A_{q}>0$ is some constant. Hence 2.21) is meaningful. Now for any $a$ such that $I_{a}>q$, 2.16-2.20 imply that 2.22 -2.24 hold.

\section{Proofs of the main theorems}

In this section, we give proofs of Theorems 1.1 and 1.2 by using the mean index identity of [WHL], Morse inequality and the index iteration theory developed by Long and his coworkers.

Following Definition 1.1 of [LoZ], we introduce

Definition 3.1. For $\alpha \in(1,2)$, we define a map $\varrho_{n}: \mathcal{H}(2 n) \rightarrow \mathbb{N} \cup\{\infty\}$ by

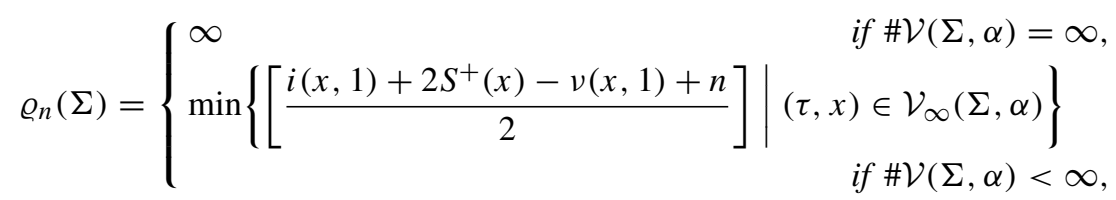

where $\mathcal{V}(\Sigma, \alpha)$ and $\mathcal{V}_{\infty}(\Sigma, \alpha)$ are the variationally visible and infinite variationally visible sets respectively given by Definition 1.4 of [LoZ] (cf. Definition 15.3.3 of [Lon4]). 
Theorem 3.2 (cf. Theorem 15.1.1 of [Lon4]). Suppose $(\tau, y) \in \mathcal{J}(\Sigma)$. Then

$$
i\left(y^{m}\right) \equiv i(m \tau, y)=i(y, m)-n, \quad v\left(y^{m}\right) \equiv v(m \tau, y)=v(y, m), \quad \forall m \in \mathbb{N},
$$

where $i(y, m)$ and $v(y, m)$ are the Maslov-type index and nullity of $(m \tau, y)$ defined by Conley, Zehnder and Long (cf. \$5.4 of [Lon4]).

Recall that for a principal $U(1)$-bundle $E \rightarrow B$, the Fadell-Rabinowitz index (cf. [FaR]) of $E$ is defined to be $\sup \left\{k \mid c_{1}(E)^{k-1} \neq 0\right\}$, where $c_{1}(E) \in H^{2}(B, \mathbb{Q})$ is the first rational Chern class. For a $U(1)$-space, i.e., a topological space $X$ with a $U(1)$-action, the FadellRabinowitz index is defined to be the index of the bundle $X \times S^{\infty} \rightarrow X \times_{U(1)} S^{\infty}$, where $S^{\infty} \rightarrow \mathbb{C} P^{\infty}$ is the universal $U(1)$-bundle.

As on p. 199 of [Eke3], choose some $\alpha \in(1,2)$ and associate with $U$ a convex function $H$ such that $H(\lambda x)=\lambda^{\alpha} H(x)$ for $\lambda \geq 0$. Consider the fixed period problem

$$
\left\{\begin{array}{l}
\dot{x}(t)=J H^{\prime}(x(t)) \\
x(1)=x(0)
\end{array}\right.
$$

Define

$$
L_{0}^{\alpha /(\alpha-1)}\left(S^{1}, \mathbb{R}^{2 n}\right)=\left\{u \in L^{\alpha /(\alpha-1)}\left(S^{1}, \mathbb{R}^{2 n}\right) \mid \int_{0}^{1} u d t=0\right\} .
$$

The corresponding Clarke-Ekeland dual action functional is defined by

$$
\Phi(u)=\int_{0}^{1}\left(\frac{1}{2} J u \cdot M u+H^{*}(-J u)\right) d t, \quad \forall u \in L_{0}^{\alpha /(\alpha-1)}\left(S^{1}, \mathbb{R}^{2 n}\right),
$$

where $M u$ is defined by $\frac{d}{d t} M u(t)=u(t)$ and $\int_{0}^{1} M u(t) d t=0$, and $H^{*}$ is the Fenchel transform of $H$ defined in $\$ 2$.

For any $\kappa \in \mathbb{R}$, we set

$$
\Phi^{\kappa-}=\left\{u \in L_{0}^{\alpha /(\alpha-1)}\left(S^{1}, \mathbb{R}^{2 n}\right) \mid \Phi(u)<\kappa\right\} .
$$

Then as on p. 218 of [Eke3], we define

$$
c_{i}=\inf \left\{\delta \in \mathbb{R} \mid \hat{I}\left(\Phi^{\delta-}\right) \geq i\right\},
$$

where $\hat{I}$ is the Fadell-Rabinowitz index given above. Then by Proposition 3 on p. 218 of [Eke3], we have

Proposition 3.3. Every $c_{i}$ is a critical value of $\Phi$. If $c_{i}=c_{j}$ for some $i<j$, then there are infinitely many geometrically distinct closed characteristics on $\Sigma$.

As in Definition 2.1, we introduce

Definition 3.4. Suppose $u$ is a nonzero critical point of $\Phi$, and $\mathcal{N}$ is an $S^{1}$-invariant open neighborhood of $S^{1} \cdot u$ such that $\operatorname{crit}(\Phi) \cap(\Lambda(u) \cap \mathcal{N})=S^{1} \cdot u$. Then the $S^{1}$-critical module of $S^{1} \cdot u$ is defined by

$$
C_{S^{1}, q}\left(\Phi, S^{1} \cdot u\right)=H_{q}\left((\Lambda(u) \cap \mathcal{N})_{S^{1}},\left(\left(\Lambda(u) \backslash S^{1} \cdot u\right) \cap \mathcal{N}\right)_{S^{1}}\right),
$$

where $\Lambda(u)=\left\{w \in L_{0}^{\alpha /(\alpha-1)}\left(S^{1}, \mathbb{R}^{2 n}\right) \mid \Phi(w) \leq \Phi(u)\right\}$. 
Comparing with Theorem 4 on p. 219 of [Eke3], we have the following

Proposition 3.5. For every $i \in \mathbb{N}$, there exists a point $u \in L_{0}^{\alpha /(\alpha-1)}\left(S^{1}, \mathbb{R}^{2 n}\right)$ such that

$$
\begin{aligned}
& \Phi^{\prime}(u)=0, \quad \Phi(u)=c_{i}, \\
& C_{S^{1}, 2(i-1)}\left(\Phi, S^{1} \cdot u\right) \neq 0 .
\end{aligned}
$$

Proof. By Lemma 8 on p. 206 of [Eke3], we can use Theorem 1.4.2 of [Cha] in the equivariant form to obtain

$$
H_{S^{1}, *}\left(\Phi^{c_{i}+\epsilon}, \Phi^{c_{i}-\epsilon}\right)=\bigoplus_{\Phi(u)=c_{i}} C_{S^{1}, *}\left(\Phi, S^{1} \cdot u\right),
$$

for $\epsilon$ small enough such that the interval $\left(c_{i}-\epsilon, c_{i}+\epsilon\right)$ contains no critical values of $\Phi$ except $c_{i}$.

Similar to p. 431 of $[\mathrm{EkH}]$, we have the exact sequence

$$
H^{2(i-1)}\left(\left(\Phi^{c_{i}+\epsilon}\right)_{S^{1}},\left(\Phi^{c_{i}-\epsilon}\right)_{S^{1}}\right) \stackrel{q^{*}}{\longrightarrow} H^{2(i-1)}\left(\left(\Phi^{c_{i}+\epsilon}\right)_{S^{1}}\right) \stackrel{p^{*}}{\longrightarrow} H^{2(i-1)}\left(\left(\Phi^{c_{i}-\epsilon}\right)_{S^{1}}\right)
$$

where $p$ and $q$ are natural inclusions. Denote by $f:\left(\Phi^{c_{i}+\epsilon}\right)_{S^{1}} \rightarrow \mathbb{C} P^{\infty}$ a classifying map and let $f^{ \pm}=\left.f\right|_{\left(\Phi^{c_{i} \pm \epsilon}\right)_{S^{1}}}$. Then clearly each $f^{ \pm}:\left(\Phi^{c_{i} \pm \epsilon}\right)_{S^{1}} \rightarrow \mathbb{C} P^{\infty}$ is a classifying map on $\left(\Phi^{c_{i} \pm \epsilon}\right)_{S^{1}}$. Let $\eta \in H^{2}\left(\mathbb{C} P^{\infty}\right)$ be the first universal Chern class.

By definition of $c_{i}$, we have $\hat{I}\left(\Phi^{c_{i}-\epsilon}\right)<i$, hence $\left(f^{-}\right)^{*}\left(\eta^{i-1}\right)=0$. Note that $p^{*}\left(f^{+}\right)^{*}\left(\eta^{i-1}\right)=\left(f^{-}\right)^{*}\left(\eta^{i-1}\right)$. Hence the exactness of 3.12 yields a $\sigma \in$ $H^{2(i-1)}\left(\left(\Phi^{c_{i}+\epsilon}\right)_{S^{1}},\left(\Phi^{c_{i}-\epsilon}\right)_{S^{1}}\right)$ such that $q^{*}(\sigma)=\left(f^{+}\right)^{*}\left(\eta^{i-1}\right)$. Since $\hat{I}\left(\Phi^{c_{i}+\epsilon}\right) \geq i$, we have $\left(f^{+}\right)^{*}\left(\eta^{i-1}\right) \neq 0$. Hence $\sigma \neq 0$, and so

$$
H_{S^{1}}^{2(i-1)}\left(\Phi^{c_{i}+\epsilon}, \Phi^{c_{i}-\epsilon}\right)=H^{2(i-1)}\left(\left(\Phi^{c_{i}+\epsilon}\right)_{S^{1}},\left(\Phi^{c_{i}-\epsilon}\right)_{S^{1}}\right) \neq 0 .
$$

Now the proposition follows from 3.11 and the universal coefficient theorem.

Proposition 3.6. Suppose $u$ is the critical point of $\Phi$ found in Proposition 3.5. Then

$$
C_{S^{1}, 2(i-1)}\left(\Psi_{a}, S^{1} \cdot u_{a}\right) \neq 0
$$

where $\Psi_{a}$ is given by 2.3 and $u_{a} \in L_{0}^{2}\left(S^{1}, \mathbb{R}^{2 n}\right)$ is its critical point corresponding to $u$ in the natural sense.

Proof. Fixing $u$, we modify the function $H$ only in a small neighborhood $\Omega$ of 0 as in [Eke1] so that the corresponding orbit of $u$ does not enter $\Omega$ and the resulting function $\widetilde{H}$ has properties similar to those in Definition 1 on p. 26 of [Eke1] with $3 / 2$ there replaced by $\alpha$. Define the dual action functional $\widetilde{\Phi}: L_{0}^{\alpha /(\alpha-1)}\left(S^{1}, \mathbb{R}^{2 n}\right) \rightarrow \mathbb{R}$ by

$$
\widetilde{\Phi}(v)=\int_{0}^{1}\left(\frac{1}{2} J v \cdot M v+\widetilde{H}^{*}(-J v)\right) d t
$$


clearly $\Phi$ and $\widetilde{\Phi}$ are $C^{1}$-close to each other. By the continuity of critical modules (cf. Theorem 8.8 of [MaW] or Theorem 1.5.6 on p. 53 of [Cha], which can be easily generalized to the equivariant case) for the $u$ in the proposition, we have

$$
C_{S^{1}, *}\left(\Phi, S^{1} \cdot u\right) \cong C_{S^{1}, *}\left(\widetilde{\Phi}, S^{1} \cdot u\right) .
$$

Using a finite-dimensional approximation as in Lemma 3.9 of [Eke1], we have

$$
C_{S^{1}, *}\left(\widetilde{\Phi}, S^{1} \cdot u\right) \cong H_{*}\left(\widetilde{\Lambda}(u) \cap D_{l} N(u),(\widetilde{\Lambda}(u) \backslash\{u\}) \cap D_{l} N(u)\right)^{\mathbb{Z}_{m}},
$$

where $\widetilde{\Lambda}(u)=\left\{w \in L_{0}^{\alpha /(\alpha-1)}\left(S^{1}, \mathbb{R}^{2 n}\right) \mid \widetilde{\Phi}(w) \leq \widetilde{\Phi}(u)\right\}$ and $D_{\iota} N(u)$ is a $\mathbb{Z}_{m}$-invariant finite-dimensional disk transversal to $S^{1} \cdot u$ at $u$ (cf. Lemma 3.9 of [WHL]), $m$ being the multiplicity of $u$.

By Lemma 3.9 of [WHL], we have

$$
C_{S^{1}, *}\left(\Psi_{a}, S^{1} \cdot u_{a}\right) \cong H_{*}\left(\Lambda_{a}\left(u_{a}\right) \cap D_{l} N\left(u_{a}\right),\left(\Lambda_{a}\left(u_{a}\right) \backslash\left\{u_{a}\right\}\right) \cap D_{l} N\left(u_{a}\right)\right)^{\mathbb{Z}_{m}} .
$$

By the construction of $H_{a}$ in [WHL], $H_{a}=\widetilde{H}$ in an $L^{\infty}$-neighborhood of $S^{1} \cdot u$. We remark here that multiplying $H$ by a constant will not affect the corresponding critical modules, i.e., the corresponding critical orbits have isomorphic critical modules. Hence we can assume $H_{a}=H$ in an $L^{\infty}$-neighborhood of $S^{1} \cdot u$. Then $\Psi_{a}$ and $\widetilde{\Phi}$ coincide in an $L^{\infty}$-neighborhood of $S^{1} \cdot u$. Note also that by Lemma 3.9 of [Eke1], the two finitedimensional approximations are actually the same. Hence we have

$$
\begin{aligned}
& H_{*}\left(\tilde{\Lambda}(u) \cap D_{l} N(u),(\tilde{\Lambda}(u) \backslash\{u\}) \cap D_{l} N(u)\right)^{\mathbb{Z}_{m}} \\
& \cong H_{*}\left(\Lambda_{a}\left(u_{a}\right) \cap D_{l} N\left(u_{a}\right),\left(\Lambda_{a}\left(u_{a}\right) \backslash\left\{u_{a}\right\}\right) \cap D_{l} N\left(u_{a}\right)\right)^{\mathbb{Z}_{m}} .
\end{aligned}
$$

Now the proposition follows from Proposition 3.5 and 3.16 - 3.18 .

Now we can give:

Proof of Theorem 1.1. By the assumption (F) at the beginning of Section 2, we let $\left\{\left(\tau_{j}, y_{j}\right)\right\}_{1 \leq j \leq k}$ be all the geometrically distinct closed characteristics on $\Sigma$, and denote by $\gamma_{j} \equiv \gamma_{y_{j}}$ the associated symplectic path of $\left(\tau_{j}, y_{j}\right)$ on $\Sigma$ for $1 \leq j \leq k$. Then by Lemma 15.2.4 of [Lon4], there exist $P_{j} \in \mathrm{Sp}(6)$ and $M_{j} \in \mathrm{Sp}(4)$ such that

$$
\gamma_{j}\left(\tau_{j}\right)=P_{j}^{-1}\left(N_{1}(1,1) \diamond M_{j}\right) P_{j}, \quad \forall 1 \leq j \leq k,
$$

where $N_{1}(1, b)=\left(\begin{array}{ll}1 & b \\ 0 & 1\end{array}\right)$ for $b \in \mathbb{R}$.

Without loss of generality, by Theorem 1.3 of [LoZ] (cf. Theorem 15.5.2 of [Lon4]), we may assume that $\left(\tau_{1}, y_{1}\right)$ has irrational mean index. Hence by Theorem 8.3.1 and Corollary 8.3.2 of [Lon4], $M_{1} \in \operatorname{Sp}(4)$ in 3.19] can be connected to $R\left(\theta_{1}\right) \diamond Q_{1}$ within

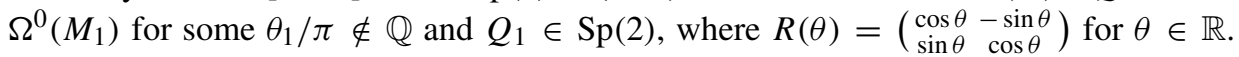
Here we use the notations from Definition 1.8.5 and Theorem 1.8.10 of [Lon4]. By Theorem 2.7 , the following identity holds:

$$
\frac{\hat{\chi}\left(y_{1}\right)}{\hat{i}\left(y_{1}\right)}+\sum_{2 \leq j \leq k} \frac{\hat{\chi}\left(y_{j}\right)}{\hat{i}\left(y_{j}\right)}=\frac{1}{2} .
$$


Now we have the following four cases according to the classification of basic norm forms (cf. Definition 1.8.9 of [Lon4]).

Case 1. $Q_{1}=R\left(\theta_{2}\right)$ with $\theta_{2} / \pi \notin \mathbb{Q}$ or $Q_{1}=D( \pm 2) \equiv\left(\begin{array}{cc} \pm 2 & 0 \\ 0 & \pm 1 / 2\end{array}\right)$.

In this case, by Theorems 8.1.6 and 8.1.7 of [Lon4], we have $v\left(y_{1}^{m}\right) \equiv 1$, i.e., $y_{1}^{m}$ is nondegenerate for all $m \in \mathbb{N}$. Hence it follows from 2.15 that $\hat{\chi}\left(y_{1}\right) \neq 0$. Now 3.20 implies that at least one of the $y_{j}$ 's for $2 \leq j \leq k$ must have irrational mean index. Hence the conclusion of the theorem holds.

Case 2. $Q_{1}=N_{1}(1, b)$ with $b= \pm 1,0$.

We have two subcases according to the value of $\hat{\chi}\left(y_{1}\right)$.

Subcase 2.1. $\hat{\chi}\left(y_{1}\right) \neq 0$.

In this case, 3.20 implies that at least one of the $y_{j}$ 's for $2 \leq j \leq k$ must have irrational mean index. Hence the desired conclusion holds.

Subcase 2.2. $\hat{\chi}\left(y_{1}\right)=0$.

Note that by Theorems 8.1.4 and 8.1.7 of [Lon4] and our Proposition 2.5, we have $K\left(y_{1}\right)=1$. Since $v\left(y_{1}\right) \leq 3$, it follows from Proposition 2.6 and 2.14 that

$$
0=\hat{\chi}\left(y_{1}\right)=(-1)^{i\left(y_{1}\right)}\left(k_{0}\left(y_{1}\right)-k_{1}\left(y_{1}\right)+k_{2}\left(y_{1}\right)\right) .
$$

By Proposition 2.6(iv), at most one of $k_{l}\left(y_{1}\right)$ for $l=0,1,2$ can be nonzero. Then 3.21) yields $k_{l}\left(y_{1}\right)=0$ for $l=0,1,2$. Hence it follows from Proposition 2.3 and Definition 2.4 that

$$
C_{S^{1}, q}\left(\Psi_{a}, S^{1} \cdot u_{1}^{p}\right)=0, \quad \forall p \in \mathbb{N}, q \in \mathbb{Z},
$$

where we denote by $u_{1}$ the critical point of $\Psi_{a}$ corresponding to $\left(\tau_{1}, y_{1}\right)$. In other words, $u_{1}^{m}$ is homologically invisible for all $m \in \mathbb{N}$.

By Propositions 3.5 and 3.6, we can replace the phrase infinite variationally visible in Definition 1.4 of [LoZ] (cf. Definition 15.3 .3 of [Lon4]) by homologically visible, and it is easy to check that all the results in [LoZ] remain true under this change. Hence by Theorem 1.3 of [LoZ] (cf. Theorem 15.5.2 of [Lon4]), at least one of the $y_{j}$ 's for $2 \leq j \leq k$ must have irrational mean index, i.e., we can forget $y_{1}$ and consider only $y_{j}$ 's for $2 \leq j \leq k$, and then apply that theorem. This proves the desired conclusion.

Case 3. $Q_{1}=N_{1}(-1,1)$.

In this case, by Theorems 8.1.4, 8.1.5 and 8.1.7 of [Lon4], we have

$$
i\left(y_{1}, m\right)=m i\left(y_{1}, 1\right)+2 E\left(\frac{m \theta_{1}}{2 \pi}\right)-2, \quad v\left(y_{1}, m\right)=1+\frac{1+(-1)^{m}}{2}, \quad \forall m \in \mathbb{N}
$$

with $i\left(y_{1}, 1\right) \in 2 \mathbb{Z}+1$. Hence $K\left(y_{1}\right)=2$ by Proposition 2.5 . Because $y_{1}$ is nondegenerate, we have $k_{l}\left(y_{1}\right)=\delta_{0}^{l}$ for all $l \in \mathbb{Z}$ by 2.11, 2.13 and Definition 2.4. By Theorem 3.2 , we have $i\left(y_{1}\right)=i\left(y_{1}, 1\right)-3 \in 2 \mathbb{Z}$ and $i\left(y_{1}^{2}\right)-i\left(y_{1}\right)=i\left(y_{1}, 2\right)-i\left(y_{1}, 1\right) \in 2 \mathbb{Z}+1$. 
Hence $k_{0}\left(y_{1}^{2}\right)=0$ by Proposition 2.6(v). Because $v\left(y_{1}^{2}\right)=2$, we have $k_{l}\left(y_{1}^{2}\right)=0$ for $l \geq 2$. Then 2.14) implies

$$
\hat{\chi}\left(y_{1}\right)=\frac{1+k_{1}\left(y_{1}^{2}\right)}{2} \neq 0 .
$$

Now (3.20 implies that at least one of the $y_{j}$ 's for $2 \leq j \leq k$ must have irrational mean index. Hence the conclusion of the theorem holds.

Case 4. $Q_{1}=N_{1}(-1, b)$ with $b=0,-1$ or $Q_{1}=R\left(\theta_{2}\right)$ with $\theta_{2} / 2 \pi=L / N \in$ $\mathbb{Q} \cap(0,1)$ with $N>1$ and $(L, N)=1$.

Note first that if $Q_{1}=N_{1}(-1, b)$ with $b=0,-1$, then Theorems 8.1.5 and 8.1.7 of [Lon4] imply that their index iteration formulae coincide with that of a rotational matrix $R(\theta)$ with $\theta=\pi$. Hence in the following we shall only consider the case $Q_{1}=R\left(\theta_{2}\right)$ with $\theta_{2} / \pi \in(0,2) \cap \mathbb{Q}$. The same argument also shows that the conclusion of the theorem is true for $Q_{1}=N_{1}(-1,-1)$.

By Theorems 8.1.4 and 8.1.7 of [Lon4], we have

$$
\begin{aligned}
i\left(y_{1}, m\right) & =m\left(i\left(y_{1}, 1\right)-1\right)+2 E\left(\frac{m \theta_{1}}{2 \pi}\right)+2 E\left(\frac{m \theta_{2}}{2 \pi}\right)-3, \\
\nu\left(y_{1}, m\right) & =3-2 \varphi\left(\frac{m \theta_{2}}{2 \pi}\right),
\end{aligned}
$$

with $i\left(y_{1}, 1\right) \in 2 \mathbb{Z}+1$ and all $m \in \mathbb{N}$. By Proposition 2.5, we have $K\left(y_{1}\right)=N$. Note that because $y_{1}^{m}$ is nondegenerate for $1 \leq m \leq N-1$, it follows that $k_{l}\left(y_{1}^{m}\right)=\delta_{0}^{l}$ for $1 \leq m \leq N-1$ by (2.11), (2.13) and Definition 2.4. By Theorem 3.2, we have $i\left(y_{1}\right)=i\left(y_{1}, 1\right)-3 \in 2 \mathbb{Z}$. Then 2.14 ) implies

$$
\hat{\chi}\left(y_{1}\right)=\frac{N-1+k_{0}\left(y_{1}^{N}\right)-k_{1}\left(y_{1}^{N}\right)+k_{2}\left(y_{1}^{N}\right)}{N} .
$$

This follows from $v\left(y_{1}^{m}\right) \leq 3$ for all $m \in \mathbb{N}$.

We have two subcases according to the value of $\hat{\chi}\left(y_{1}\right)$.

Subcase 4.1. $\hat{\chi}\left(y_{1}\right) \neq 0$.

In this subcase, 3.20 implies that at least one of the $y_{j}$ 's for $2 \leq j \leq k$ must have irrational mean index. Hence the conclusion holds.

Subcase 4.2. $\hat{\chi}\left(y_{1}\right)=0$.

In this subcase, it follows from 3.25 and Proposition 2.6(iv) that

$$
k_{1}\left(y_{1}^{N}\right)=N-1>0 .
$$

Using the common index jump theorem (Theorems 4.3 and 4.4 of [LoZ], Theorems 11.2.1 and 11.2.2 of [Lon4]), we obtain some $\left(T, m_{1}, \ldots, m_{k}\right) \in \mathbb{N}^{k+1}$ such that $m_{1} \theta_{2} / \pi \in \mathbb{Z}$ 
(cf. (11.2.18) of [Lon4]) and the following hold by (11.2.6), (11.2.7) and (11.2.26) of [Lon4]:

$$
\begin{aligned}
i\left(y_{j}, 2 m_{j}\right) & \geq 2 T-e\left(\gamma_{j}\left(\tau_{j}\right)\right) / 2 \\
i\left(y_{j}, 2 m_{j}\right)+v\left(y_{j}, 2 m_{j}\right) & \leq 2 T+e\left(\gamma_{j}\left(\tau_{j}\right)\right) / 2-1, \\
i\left(y_{j}, 2 m_{j}+1\right) & =2 T+i\left(y_{j}, 1\right), \\
i\left(y_{j}, 2 m_{j}-1\right)+v\left(y_{j}, 2 m_{j}-1\right) & =2 T-\left(i\left(y_{j}, 1\right)+2 S_{\gamma_{j}\left(\tau_{j}\right)}^{+}(1)-v\left(y_{j}, 1\right)\right) .
\end{aligned}
$$

By p. 340 of [Lon4], we have

$$
\begin{aligned}
2 S_{\gamma_{j}\left(\tau_{j}\right)}^{+}(1)-v\left(y_{j}, 1\right) & =2 S_{N_{1}(1,1)}^{+}(1)-v_{1}\left(N_{1}(1,1)\right)+2 S_{M_{j}}^{+}(1)-v_{1}\left(M_{j}\right) \\
& =1+2 S_{M_{j}}^{+}(1)-v_{1}\left(M_{j}\right) \\
& \geq-1, \quad 1 \leq j \leq k
\end{aligned}
$$

In the last inequality, we have used the fact that the worst case for $2 S_{M_{j}}^{+}(1)-v_{1}\left(M_{j}\right)$ happens when $M_{j}=N_{1}(1,-1)^{\diamond 2}$, which gives the lower bound -2 .

By Corollary 15.1.4 of [Lon4], we have $i\left(y_{j}, 1\right) \geq 3$ for $1 \leq j \leq k$. Note that $e\left(\gamma_{j}\left(\tau_{j}\right)\right) \leq 6$ for $1 \leq j \leq k$. Hence Theorem 10.2.4 of [Lon4] yields

$$
\begin{aligned}
i\left(y_{j}, m\right)+v\left(y_{j}, m\right) & \leq i\left(y_{j}, m+1\right)-i\left(y_{j}, 1\right)+e\left(\gamma_{j}\left(\tau_{j}\right)\right) / 2-1 \\
& \leq i\left(y_{j}, m+1\right)-1, \quad \forall m \in \mathbb{N}, 1 \leq j \leq k .
\end{aligned}
$$

In particular, we have

$$
i\left(y_{j}, m\right)<i\left(y_{j}, m+1\right), \quad \forall m \in \mathbb{N}, 1 \leq j \leq k .
$$

Now 3.27 -3.30 become

$$
\begin{aligned}
& i\left(y_{j}, 2 m_{j}\right) \geq 2 T-3, \\
& i\left(y_{j}, 2 m_{j}\right)+v\left(y_{j}, 2 m_{j}\right)-1 \leq 2 T+1, \\
& i\left(y_{j}, 2 m_{j}+m\right) \geq 2 T+3, \quad \forall m \geq 1, \\
& i\left(y_{j}, 2 m_{j}-m\right)+v\left(y_{j}, 2 m_{j}-m\right)-1 \leq 2 T-3, \quad \forall m \geq 1,
\end{aligned}
$$

where $1 \leq j \leq k$. By Proposition 2.3, we have

$$
C_{S^{1}, q}\left(\Psi_{a}, S^{1} \cdot u_{1}^{2 m_{1}}\right)=\delta_{i\left(u_{1}^{2 m_{1}}\right)+1}^{q} \mathbb{Q}^{k_{1}\left(y_{1}^{N}\right)}=\delta_{i\left(u_{1}^{2 m_{1}}\right)+1}^{q} \mathbb{Q}^{N-1} .
$$

Note that by Theorem 3.2,

$$
i\left(y_{j}^{m}\right)=i\left(y_{j}, m\right)-3, \quad \forall m \in \mathbb{N}, 1 \leq j \leq k .
$$


Hence 3.23 implies that $i\left(y_{1}^{m}\right)$ is even for all $m \in \mathbb{N}$. This together with 3.35-3.38 and Proposition 2.3 yield

$$
\begin{array}{ll}
C_{S^{1}, 2 T-2}\left(\Psi_{a}, S^{1} \cdot u_{1}^{m}\right)=0, & \forall m \in \mathbb{N}, \\
C_{S^{1}, 2 T-4}\left(\Psi_{a}, S^{1} \cdot u_{1}^{m}\right)=0, & \forall m \in \mathbb{N}, \\
C_{S^{1}, 2 T-2}\left(\Psi_{a}, S^{1} \cdot u_{j}^{m}\right)=0, & \forall m \neq 2 m_{j}, 2 \leq j \leq k, \\
C_{S^{1}, 2 T-4}\left(\Psi_{a}, S^{1} \cdot u_{j}^{m}\right)=0, & \forall m \neq 2 m_{j}, 2 \leq j \leq k .
\end{array}
$$

In fact, by 3.35, , 3.36 and 3.38 for $1 \leq j \leq k$, we have $i\left(u_{j}^{m}\right)=i\left(y_{j}^{m}\right) \geq 2 T$ for all $m>2 m_{j}$ and $i\left(u_{j}^{m}\right)+v\left(u_{j}^{m}\right)-1=i\left(y_{j}^{m}\right)+v\left(y_{j}^{m}\right)-1 \leq 2 T-6$ for all $m<2 m_{j}$. Thus 3.41 -3.42 hold and 3.39 -3.40 hold for $m \neq 2 m_{1}$ by Proposition 2.3. Since $i\left(y_{1}^{2 m_{1}}\right)$ is even, by 3.37, 3.39)-3.40) also hold for $m=2 m_{1}$.

Thus by Propositions 3.5 and 3.6 we can find $p, q \in\{2, \ldots, k\}$ such that

$$
\begin{array}{lll}
\Phi^{\prime}\left(u_{p}^{2 m_{p}}\right)=0, & \Phi\left(u_{p}^{2 m_{p}}\right)=c_{T-1}, & C_{S^{1}, 2 T-4}\left(\Psi_{a}, S^{1} \cdot u_{p}^{2 m_{p}}\right) \neq 0, \\
\Phi^{\prime}\left(u_{q}^{2 m_{q}}\right)=0, & \Phi\left(u_{q}^{2 m_{q}}\right)=c_{T}, \quad C_{S^{1}, 2 T-2}\left(\Psi_{a}, S^{1} \cdot u_{q}^{2 m_{q}}\right) \neq 0,
\end{array}
$$

where we also denote by $u_{p}^{2 m_{p}}$ and $u_{q}^{2 m_{q}}$ the corresponding critical points of $\Phi$; this will not lead to confusion.

Note that by assumption (F) and Proposition 3.3, we have $c_{T-1}<c_{T}$. Hence $p \neq q$ by (3.43) and (3.44). Then the proof of Lemma 3.1 in [LoZ] (cf. Lemma 15.3.5 of [Lon4]) yields

$$
\hat{i}\left(y_{p}, 2 m_{p}\right)<\hat{i}\left(y_{q}, 2 m_{q}\right) .
$$

Now if both $\hat{i}\left(y_{p}\right) \in \mathbb{Q}$ and $\hat{i}\left(y_{q}\right) \in \mathbb{Q}$, then the proof of Theorem 5.3 in [LoZ] (cf. Theorem 15.5.2 of [Lon4]) yields

$$
\hat{i}\left(y_{p}, 2 m_{p}\right)=\hat{i}\left(y_{q}, 2 m_{q}\right) .
$$

Note that we may first choose $T$ such that $T / M \hat{i}\left(y_{j}\right) \in \mathbb{N}$ for all $\hat{i}\left(y_{j}\right) \in \mathbb{Q}$ and then use the proof of Theorem 5.3 in [LoZ]. Here $M$ is the least integer in $\mathbb{N}$ that satisfies $M \theta / \pi \in \mathbb{Z}$ whenever $e^{\sqrt{-1} \theta} \in \sigma\left(\gamma_{j}\left(\tau_{j}\right)\right)$ and $\theta / \pi \in \mathbb{Q}$ for some $1 \leq j \leq k$. Hence either $\hat{i}\left(y_{p}\right) \notin \mathbb{Q}$ or $\hat{i}\left(y_{q}\right) \notin \mathbb{Q}$. This together with $\hat{i}\left(y_{1}\right) \notin \mathbb{Q}$ and $p, q \neq 1$ proves the theorem.

Proof of Theorem 1.2. We denote by $\left\{\left(\tau_{j}, y_{j}\right)\right\}_{1 \leq j \leq 3}$ the three geometrically distinct closed characteristics on $\Sigma$, and by $\gamma_{j} \equiv \gamma_{y_{j}}$ the associated symplectic path of $\left(\tau_{j}, y_{j}\right)$ on $\Sigma$ for $1 \leq j \leq 3$. Then as in the proof of Theorem 1.1, there exist $P_{j} \in \operatorname{Sp}(6)$ and $M_{j} \in \mathrm{Sp}(4)$ such that

$$
\gamma_{j}\left(\tau_{j}\right)=P_{j}^{-1}\left(N_{1}(1,1) \diamond M_{j}\right) P_{j}, \quad \forall 1 \leq j \leq 3 .
$$

As on p. 356 of [LoZ], if there is no $\left(\tau_{j}, y_{j}\right)$ with $M_{j}=N_{1}(1,-1)^{\diamond 2}$ and $i\left(y_{j}, 1\right)=3$ in $\mathcal{V}_{\infty}(\Sigma, \alpha)$, then $\varrho_{n}(\Sigma)=3$. Hence we can use Theorem 1.4 of [LoZ] (Theorem 15.5.2 
of [Lon4]) to obtain the existence of at least two elliptic closed characteristics. This proves the assertion of the theorem.

It remains to show that if there exists a $\left(\tau_{j}, y_{j}\right)$ with $M_{j}=N_{1}(1,-1)^{\diamond 2}$ and $i\left(y_{j}, 1\right)=3$ in $\mathcal{V}_{\infty}(\Sigma, \alpha)$, then we have at least two elliptic closed characteristics. We may assume $M_{1}=N_{1}(1,-1)^{\diamond 2}$ and $i\left(y_{1}, 1\right)=3$ without loss of generality. Note that $\left(\tau_{1}, y_{1}\right)$ has rational mean index by Theorem 8.3.1 of [Lon4] and Theorem 3.2.

By Theorem 1.3 of [LoZ], we may assume that $\left(\tau_{2}, y_{2}\right)$ has irrational mean index. Hence by Theorem 8.3.1 and Corollary 8.3.2 of [Lon4], $M_{2} \in \mathrm{Sp}(4)$ in (3.46) can be connected to $R\left(\theta_{2}\right) \diamond Q_{2}$ within $\Omega^{0}\left(M_{2}\right)$ for some $\theta_{2} / \pi \in \mathbb{R} \backslash \mathbb{Q}$ and $Q_{2} \in \mathrm{Sp}(2)$, where

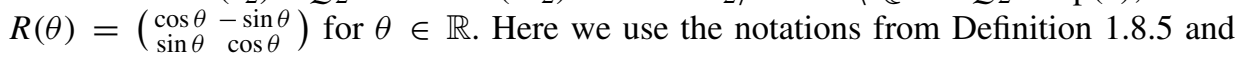
Theorem 1.8.10 of [Lon4]. By Theorem 2.7,

$$
\frac{\hat{\chi}\left(y_{1}\right)}{\hat{i}\left(y_{1}\right)}+\frac{\hat{\chi}\left(y_{2}\right)}{\hat{i}\left(y_{2}\right)}+\frac{\hat{\chi}\left(y_{3}\right)}{\hat{i}\left(y_{3}\right)}=\frac{1}{2} .
$$

Now if $Q_{2}$ is not hyperbolic, then both $\left(\tau_{1}, y_{1}\right)$ and $\left(\tau_{2}, y_{2}\right)$ are elliptic, so the conclusion of the theorem holds.

Hence it remains to consider the case where $Q_{2}$ is hyperbolic. Clearly $\left(\tau_{2}, y_{2}\right)$ is nondegenerate, so it follows from 2.15 that $\hat{\chi}\left(y_{2}\right) \neq 0$. Hence 3.47 implies that $\hat{i}\left(y_{3}\right) \in$ $\mathbb{R} \backslash \mathbb{Q}$. Now by Theorem 8.3.1 and Corollary 8.3.2 of [Lon4], $M_{3} \in \operatorname{Sp}(4)$ in (3.46) can be connected to $R\left(\theta_{3}\right) \diamond Q_{3}$ within $\Omega^{0}\left(M_{3}\right)$ for some $\theta_{3} / \pi \in \mathbb{R} \backslash \mathbb{Q}$ and $Q_{3} \in \operatorname{Sp}(2)$. By the same reason as above, it suffices to consider the case where $Q_{3}$ is hyperbolic.

Combining all the above, the only case we need to kick off is that

$M_{1}=N_{1}(1,-1)^{\diamond 2}, \quad i\left(y_{1}, 1\right)=3, \quad M_{2}=R\left(\theta_{2}\right) \diamond Q_{2}, \quad M_{3}=R\left(\theta_{3}\right) \diamond Q_{3}$,

where both $Q_{2}$ and $Q_{3}$ are hyperbolic. Then by Theorem 8.3.1 of [Lon4] and Theorem 3.2 , we have

$\begin{array}{lll}i\left(y_{1}^{m}\right)=m\left(i\left(y_{1}, 1\right)+1\right)-4=4 m-4, & v\left(y_{1}^{m}\right)=3, & \forall m \in \mathbb{N}, \\ i\left(y_{j}^{m}\right)=m\left(i\left(y_{j}\right)+3\right)+2 E\left(\frac{m \theta_{j}}{2 \pi}\right)-5, & v\left(y_{j}^{m}\right)=1, & \forall m \in \mathbb{N}, j=2,3 .\end{array}$

By Proposition 2.5, we have $K\left(y_{1}\right)=1$. Note that $i\left(y_{1}\right)=i\left(y_{1}, 1\right)-3=0$ by Theorem 3.2. Hence Proposition 2.6, 2.14 and 2.15) imply

$$
\begin{aligned}
& \hat{\chi}\left(y_{1}\right) \leq 1, \quad \hat{\chi}\left(y_{1}\right) \in \mathbb{Z}, \\
& \hat{\chi}\left(y_{j}\right)=\left\{\begin{array}{ll}
-1 & \text { if } i\left(y_{j}\right) \in 2 \mathbb{N}_{0}+1, \\
1 / 2 & \text { if } i\left(y_{j}\right) \in 2 \mathbb{N}_{0},
\end{array} \quad j=1,2 .\right.
\end{aligned}
$$

By (3.49) and 3.50), we have

$$
\begin{aligned}
& \hat{i}\left(y_{1}\right)=4, \\
& \hat{i}\left(y_{j}\right)=i\left(y_{j}\right)+3+\theta_{j} / \pi>3, \quad j=2,3 .
\end{aligned}
$$


By 3.51-3.54, in order to make 3.47) hold, we must have

$$
\begin{aligned}
\hat{\chi}\left(y_{1}\right) & =1, \\
i\left(y_{j}\right) & \in 2 \mathbb{N}_{0}, \quad j=2,3 .
\end{aligned}
$$

In fact, by 3.52 and 3.54, we have

$$
\frac{\hat{\chi}\left(y_{2}\right)}{\hat{i}\left(y_{2}\right)}+\frac{\hat{\chi}\left(y_{3}\right)}{\hat{i}\left(y_{3}\right)}<\frac{1}{6}+\frac{1}{6}<\frac{1}{2} .
$$

Thus to make 3.47 hold, we must have $\hat{\chi}\left(y_{1}\right) / \hat{i}\left(y_{1}\right)>0$. Hence 3.55 follows from (3.51). Now if $i\left(y_{2}\right) \in 2 \mathbb{N}_{0}+1$ or $i\left(y_{3}\right) \in 2 \mathbb{N}_{0}+1$, then by 3.52, we have

$$
\frac{\hat{\chi}\left(y_{1}\right)}{\hat{i}\left(y_{1}\right)}+\frac{\hat{\chi}\left(y_{2}\right)}{\hat{i}\left(y_{2}\right)}+\frac{\hat{\chi}\left(y_{3}\right)}{\hat{i}\left(y_{3}\right)}<\frac{1}{4}+\frac{1}{6}<\frac{1}{2} \text {. }
$$

Hence (3.56) must hold.

By 2.14, 3.49 and 3.55, we have $1=\hat{\chi}\left(y_{1}\right)=k_{0}\left(y_{1}\right)-k_{1}\left(y_{1}\right)+k_{2}\left(y_{1}\right)$. Since $v\left(y_{1}\right)=3$, by Proposition 2.6, only one of $k_{0}\left(y_{1}\right), k_{1}\left(y_{1}\right), k_{2}\left(y_{1}\right)$ can be nonzero. Hence we obtain

$$
k_{1}\left(y_{1}\right)=0, \quad k_{0}\left(y_{1}\right)+k_{2}\left(y_{1}\right)=1 .
$$

By Proposition 2.3, we have

$$
C_{S^{1}, q}\left(\Psi_{a}, S^{1} \cdot u_{j}^{p}\right)=0, \quad \forall p \in \mathbb{N}, q \in 2 \mathbb{Z}+1,1 \leq j \leq 3 .
$$

In fact, by 3.49 , we have $i\left(y_{1}^{m}\right) \in 2 \mathbb{N}$ for all $m \in \mathbb{N}$. Thus 3.58 holds for $j=1$ by 2.11, 3.57) and Definition 2.4. By 3.50, and 3.56, for $j=2,3$, we have $i\left(y_{j}^{m}\right) \in 2 \mathbb{N}$ when $m \in 2 \mathbb{N}_{0}+1$ and $i\left(y_{j}^{m}\right) \in 2 \mathbb{N}_{0}+1$ when $m \in 2 \mathbb{N}$. In particular, all $y_{j}^{m}$ are nondegenerate for $m \in \mathbb{N}$ and $j=2,3$. Thus (3.58) holds for $j=2,3$ by 2.13.

Note that 3.58 implies

$$
M_{q}=0, \quad \forall q \in 2 \mathbb{Z}+1
$$

Together with the Morse inequality of Theorem 2.8, this yields

$$
-M_{2 k}-\cdots-M_{2}-M_{0} \geq-b_{2 k}-\cdots-b_{2}-b_{0} .
$$

Thus by the Morse inequality again,

$$
b_{2 k}+\cdots+b_{2}+b_{0} \geq M_{2 k}+\cdots+M_{2}+M_{0} \geq b_{2 k}+\cdots+b_{2}+b_{0}
$$

for all $k \geq 0$. Therefore we obtain

$$
M_{q}=b_{q}, \quad \forall q \in \mathbb{Z} .
$$

By (3.57), we have two cases according to the values of $k_{l}\left(y_{1}\right) \mathrm{s}$.

Case 1. $k_{0}\left(y_{1}\right)=1$ and $k_{2}\left(y_{1}\right)=0$. 
In this case, by Propositions 2.3, 2.5 and Definition 2.4, we have

$$
\operatorname{dim} C_{S^{1}, q}\left(\Psi_{a}, S^{1} \cdot u_{1}^{m}\right)=\delta_{4 m-4}^{q}, \quad \forall m \in \mathbb{N}, q \in \mathbb{Z} .
$$

Then by (3.60) and 2.22), we must have

$$
C_{S^{1}, 4 m-4}\left(\Psi_{a}, S^{1} \cdot u_{j}^{p}\right)=0, \quad \forall p, m \in \mathbb{N}, j=2,3 .
$$

By (3.60) and 2.22) again, $M_{2}=b_{2}=1$ implies

$$
C \equiv C_{S^{1}, 2}\left(\Psi_{a}, S^{1} \cdot u_{j}^{p}\right)=\mathbb{Q},
$$

for some $p \in \mathbb{N}$ and $j=2$ or 3 . If $p \geq 2$, by 3.50, we have

$$
i\left(y_{j}^{p}\right) \geq 3 p+2 E\left(\frac{p \theta_{j}}{2 \pi}\right)-5 \geq 3 .
$$

Thus $C=0$ by Proposition 2.3. Hence $p=1$. Without loss of generality, we assume $j=2$. Then by Proposition 2.3 and 3.63 , we have

$$
i\left(y_{2}\right)=2 \text {. }
$$

Then by 3.50$)$, we have

$$
i\left(y_{2}^{m}\right) \geq 7, \quad \forall m \geq 2 .
$$

By (3.60) and 2.22), $M_{6}=b_{6}=1$ implies

$$
C_{S^{1}, 6}\left(\Psi_{a}, S^{1} \cdot u_{j}^{p}\right)=\mathbb{Q}
$$

for some $p \in \mathbb{N}$ and $j=2$ or 3. By (3.65) and (3.66), we have $j \neq 2$, i.e., $j=3$. We must have $p=1$. In fact, by $\left(3.61\right.$ and (3.63), $y_{1}^{m}$ and $y_{2}^{n}$ already contribute a 1 to $M_{q}$ for $q=0,2,4$. Hence by 2.22), (3.60) and (3.56), we have $i\left(y_{3}\right) \geq 6$, and so $i\left(y_{3}^{m}\right) \geq 15$ by (3.50) for $m \geq 2$. Thus $p=1$ follows from Proposition 2.3. Now we have

$$
i\left(y_{3}\right)=6 .
$$

Hence by (3.53) and 3.55) for $y_{1}$, and 3.50, 3.52, 3.65) and 3.68) for $y_{2}$ and $y_{3}$, we have

$$
\frac{\hat{\chi}\left(y_{1}\right)}{\hat{i}\left(y_{1}\right)}+\frac{\hat{\chi}\left(y_{2}\right)}{\hat{i}\left(y_{2}\right)}+\frac{\hat{\chi}\left(y_{3}\right)}{\hat{i}\left(y_{3}\right)}=\frac{1}{4}+\frac{1}{2\left(5+\theta_{2} / \pi\right)}+\frac{1}{2\left(9+\theta_{3} / \pi\right)}<\frac{1}{2} .
$$

This contradicts 3.47) and proves Case 1.

Case 2. $k_{0}\left(y_{1}\right)=0$ and $k_{2}\left(y_{1}\right)=1$.

The study of this case is similar to that of Case 1. Thus we are rather sketchy here.

In this case, by Proposition 2.3 and Definition 2.4, we have

$$
\operatorname{dim} C_{S^{1}, q}\left(\Psi_{a}, S^{1} \cdot u_{1}^{m}\right)=\delta_{4 m-2}^{q}, \quad \forall m \in \mathbb{N}, q \in \mathbb{Z} .
$$


Hence by 3.60 and 2.22, we must have

$$
C_{S^{1}, 4 m-2}\left(\Psi_{a}, S^{1} \cdot u_{j}^{p}\right)=0, \quad \forall p, m \in \mathbb{N}, j=2,3 .
$$

By 3.69), 3.60) and 2.22, $M_{0}=b_{0}=1$ implies

$$
C_{S^{1}, 0}\left(\Psi_{a}, S^{1} \cdot u_{j}^{p}\right)=\mathbb{Q}
$$

for some $p \in \mathbb{N}$ and $j=2$ or 3. By (3.64), we have $p=1$. Without loss of generality, we assume $j=2$. Then by Proposition 2.3 and 3.50 , we have

$$
i\left(y_{2}\right)=0, \quad i\left(y_{2}^{m}\right) \geq 6, \quad \forall m \geq 3 .
$$

By (3.60) and 2.22, $M_{4}=b_{4}=1$ implies

$$
C_{S^{1}, 4}\left(\Psi_{a}, S^{1} \cdot u_{j}^{p}\right)=\mathbb{Q}
$$

for some $p \in \mathbb{N}$ and $j=2$ or 3. By 3.69) and (3.72), as in the verification of 3.68, we have $j=3$ and $p=1$. Then by Proposition 2.3, we have

$$
i\left(y_{3}\right)=4 \text {. }
$$

Hence by (3.53) and 3.55) for $y_{1}$, and 3.50, 3.52, 3.72) and 3.74) for $y_{2}$ and $y_{3}$, we have

$$
\frac{\hat{\chi}\left(y_{1}\right)}{\hat{i}\left(y_{1}\right)}+\frac{\hat{\chi}\left(y_{2}\right)}{\hat{i}\left(y_{2}\right)}+\frac{\hat{\chi}\left(y_{3}\right)}{\hat{i}\left(y_{3}\right)}=\frac{1}{4}+\frac{1}{2\left(3+\theta_{2} / \pi\right)}+\frac{1}{2\left(7+\theta_{3} / \pi\right)}<\frac{1}{2} .
$$

This contradicts (3.47) and proves Case 2 and therefore the whole theorem.

Acknowledgments. I would like to sincerely thank my Ph.D. thesis advisor, Professor Yiming Long, for introducing me to Hamiltonian dynamics and for his valuable help and encouragement during the writing of this paper. Working with him is indeed enjoyable. I would also like to thank the referee for his/her careful reading and valuable comments and suggestions.

This research was partially supported NNSF No. 10801002, CPSF No. 200801021 and LMAM in Peking University.

\section{References}

[BaL] Bangert, V., Long, Y.: The existence of two closed geodesics on every Finsler 2-sphere. math.SG/0709.1243

[Cha] Chang, K. C., Infinite Dimensional Morse Theory and Multiple Solution Problems. Birkhäuser, Boston (1993) Zbl 0779.58005 MR 1196690

[CoZ] Conley, C., Zehnder, E.: Morse-type index theory for flows and periodic solutions for Hamiltonian equations. Comm. Pure Appl. Math. 37, 207-253 (1984) Zbl 0559.58019 MR 0733717

[DDE] Dell'Antonio, G., D'Onofrio, B., Ekeland, I.: Les systèmes hamiltoniens convexes et pairs ne sont pas ergodiques en général. C. R. Acad. Sci. Paris Sér. I 315, 1413-1415 (1992) Zbl 0768.70014 MR 1199013

[Eke1] Ekeland, I.: Une théorie de Morse pour les systèmes hamiltoniens convexes. Ann. Inst. H. Poincaré Anal. Non Linéaire 1, 19-78 (1984) Zbl 0537.58018 MR 0738494 
[Eke2] Ekeland, I.: An index theory for periodic solutions of convex Hamiltonian systems. In: Nonlinear Functional Analysis and Its Applications, Proc. Sympos. Pure Math. 45, Amer. Math. Soc., 395-423 (1986) Zbl 0596.34023 MR 0843575

[Eke3] Ekeland, I.: Convexity Methods in Hamiltonian Mechanics. Springer, Berlin (1990) Zbl 0707.70003 MR 1051888

[EkH] Ekeland, I., Hofer, H.: Convex Hamiltonian energy surfaces and their closed trajectories. Comm. Math. Phys. 113, 419-467 (1987) Zbl 0641.58038 MR 0925924

[EkL] Ekeland, I., Lassoued, L.: Multiplicité des trajectoires fermées de systèmes hamiltoniens convexes. Ann. Inst. H. Poincaré Anal. Non Linéaire 4, 307-335 (1987) Zbl 0633.58034 MR 0917740

[FaR] Fadell, E., Rabinowitz, P.: Generalized cohomological index theories for Lie group actions with an application to bifurcation questions for Hamiltonian systems. Invent. Math. 45, 139-174 (1978) Zbl 0403.57001 MR 0478189

[GrM] Gromoll, D., Meyer, W.: On differentiable functions with isolated critical points. Topology 8, 361-369 (1969) Zbl 0212.28903 MR 0246329

[HWZ] Hofer, H., Wysocki, K., Zehnder, E.: The dynamics on three-dimensional strictly convex energy surfaces. Ann. of Math. 148, 197-289 (1998) Zbl 0944.37031 MR 1652928

[LLZ] Liu, C., Long, Y., Zhu, C.: Multiplicity of closed characteristics on symmetric convex hypersurfaces in $\mathbb{R}^{2 n}$. Math. Ann. 323, 201-215 (2002) Zbl 1005.37030 MR 1913039

[Lon1] Long, Y., Maslov-type index, degenerate critical points, and asymptotically linear Hamiltonian systems. Sci. China Ser. A 33, 1409-1419 (1990), Zbl 0736.58022 MR 1090484

[Lon2] Long, Y.: Hyperbolic closed characteristics on compact convex smooth hypersurfaces in $\mathbb{R}^{2 n}$. J. Differential Equations 150, 227-249 (1998), Zbl 0915.58032 MR 1658613

[Lon3] Long, Y.: Precise iteration formulae of the Maslov-type index theory and ellipticity of closed characteristics. Adv. Math. 154, 76-131 (2000), Zbl 0970.37013 MR 1780096

[Lon4] Long, Y.: Index Theory for Symplectic Paths with Applications. Progr. Math. 207, Birkhäuser, Basel (2002) Zbl 1012.37012 MR 1898560

[Lon5] Long, Y.: Index iteration theory for symplectic paths with applications to nonlinear Hamiltonian systems. In: Proc. Int. Congress Math. 2002, Vol. II, Higher Edu. Press, Beijing, 303-313 (2002) Zbl 1036.37020 MR 1957042

[Lon6] Long, Y.: Index iteration theory for symplectic paths and multiple periodic solution orbits. Frontiers Math. China 1, 178-200 (2006) MR 2221070

[LoW] Long, Y., Wang, W.: Stability of closed characteristics on compact convex hypersurfaces. In: Inspired by S. S. Chern, ed. by P. Griffiths, Nankai Tracts Math. 11, World Sci., 313333 (2006). Zbl pre05234303 MR 2313339

[LZe] Long, Y., Zehnder, E.: Morse-theory for forced oscillations of asymptotically linear Hamiltonian systems. In: Stochastic Processes, Physics and Geometry (Ancona and Locarno, 1988), S. Albeverio et al. (eds.), World Sci., 528-563 (1990) MR 1124230

[LoZ] Long, Y., Zhu, C.: Closed characteristics on compact convex hypersurfaces in $\mathbb{R}^{2 n}$. Ann. of Math. 155, 317-368 (2002) Zbl 1028.53003 MR 1906590

[MaW] Mawhin, J., Willem, M.: Critical Point Theory and Hamiltonian Systems. Springer, New York (1989) Zbl 0676.58017 MR 0982267

[Rab] Rabinowitz, P. H., Periodic solutions of Hamiltonian systems. Comm. Pure Appl. Math. 31, 157-184 (1978) Zbl 0358.70014 MR 0467823

[Szu] Szulkin, A., Morse theory and existence of periodic solutions of convex Hamiltonian systems. Bull. Soc. Math. France 116, 171-197 (1988) Zbl 0669.58004 MR 0971559

[Vit] Viterbo, C., Equivariant Morse theory for starshaped Hamiltonian systems. Trans. Amer. Math. Soc. 311, 621-655 (1989) Zbl 0676.58030 MR 0978370 
[WHL] Wang, W., Hu, X., Long, Y.: Resonance identity, stability, and multiplicity of closed characteristics on compact convex hypersurfaces. Duke Math. J. 139, 411-462 (2007) Zbl 1139.58007 MR 2350849

[Wei] Weinstein, A., Periodic orbits for convex Hamiltonian systems. Ann. of Math. 108, 507518 (1978) Zbl 0403.58001 MR 0512430 